\title{
Giant myelolipomas and inadvertent bilateral adrenalectomy in classic congenital adrenal hyperplasia
}

\author{
Geetanjali Kale, Elaine M Pelley and Dawn Belt Davis \\ Division of Endocrinology, Diabetes, and Metabolism, Department of Medicine, University of Wisconsin, Madison, \\ Wisconsin, USA
}

Correspondence

should be addressed

to D B Davis

Email

dbd@medicine.wisc.edu

\section{Summary}

Myelolipomas have been reported in patients with congenital adrenal hyperplasia (CAH). ACTH excess, as seen with non-adherence to glucocorticoid therapy, may be responsible for tumor development. We report a case of a 51-year-old man with classic salt-wasting CAH managed on prednisone $7.5 \mathrm{mg}$ daily and fludrocortisone who presented with chronic back pain and was found to have giant bilateral retroperitoneal masses. On computed tomography (CT) imaging, the masses were heterogeneous, but contained predominantly low-density fat attenuation. The tumors were resected due to concern for malignancy and mass symptoms. Pathologic examination identified both retroperitoneal masses as myelolipomas. The left tumor was $34 \times 20 \times 13 \mathrm{~cm}$ and weighed $4.7 \mathrm{~kg}$ and the right tumor was $20 \mathrm{~cm}$ in the largest dimension. Adrenal tissue was present in the specimen. The patient reported long-term compliance with glucocorticoid treatment. However, no biochemical monitoring of ACTH levels had occurred. Therefore, it is unclear if ACTH excess contributed to the development of these large tumors in this patient. It was presumed that both adrenal glands were inadvertently removed during surgery and the patient was treated with physiologic replacement doses of hydrocortisone and fludrocortisone postoperatively. In this case, the bilateral adrenalectomy was inadvertent. However, adrenalectomy can be considered as a treatment option in patients with classical CAH under certain circumstances to avoid complications of glucocorticoid excess.

\section{Learning points:}

- Myelolipomas should be considered in the differential diagnosis of adrenal or retroperitoneal masses in patients with $\mathrm{CAH}$.

- On CT imaging, myelolipomas are seen as heterogeneous masses with low-density mature fat interspersed with more dense myeloid tissue.

- Myelolipomas are usually unilateral and measure $<4 \mathrm{~cm}$; however, very large and bilateral tumors have been reported.

- Treatment of CAH typically involves using supraphysiologic doses of glucocorticoid to suppress adrenal hyperandrogenism. Bilateral adrenalectomy is an alternative treatment option in patients with CAH.

- There is an association between ACTH excess and increased incidence of adrenal myelolipoma but the direct causal link remains to be established. 


\section{Background}

Myelolipomas are typically benign unilateral adrenal neoplasms measuring $<4 \mathrm{~cm}$. The tumors are comprised of adipose and myeloid tissue and are biochemically inert. Their incidence is $\sim 0.08-0.4 \%$ in the general population. They show no gender predilection and typically occur in the fifth to seventh decade of life (1). With the advent of modern imaging techniques, they are most commonly incidental findings (2) but can rarely present with acute abdominal pain due to torsion, infection, or hemorrhage. Computed tomography (CT) is the most sensitive and specific imaging modality for diagnosing myelolipomas. Myelolipomas appear as heterogeneous masses on CT imaging consisting of very low-density adipose tissue (usually $<-30$ Hounsfield units (HU)) interspersed with myeloid tissue of relatively higher density. Although adrenal adenomas may also appear as low-density lesions, they are typically more dense and homogeneous than myelolipomas (3).

An increased frequency of adrenal tumors, particularly myelolipomas, has been reported in patients with congenital adrenal hyperplasia (CAH). One study found a $4 \%$ incidence of myelolipoma in patients with CAH (4). Rarely, very large bilateral myelolipomas have been reported in $\mathrm{CAH}$, particularly in the presence of nonadherence to glucocorticoid therapy. It has been postulated that adrenocorticotropic hormone (ACTH) excess stimulates tumor growth (5) (6).

Treatment of classic salt-wasting $\mathrm{CAH}$ due to 21-hydroxylase deficiency requires lifelong glucocorticoid and mineralocorticoid replacement. Glucocorticoid therapy provides hormone replacement and reduces adrenal androgen production. The importance of androgen suppression in adult men not desiring fertility is unclear. Optimal dosing of glucocorticoids in $\mathrm{CAH}$ is challenging, and the consequences of prolonged glucocorticoid excess can be significant, including weight gain, metabolic disturbances, and bone loss. Studies on bilateral adrenalectomy as an alternate treatment option in CAH are limited, and this treatment has typically been restricted to patients who are refractory to medical therapy.

\section{Case presentation}

A 51-year-old male with a diagnosis of classic salt-wasting CAH since infancy had been treated with prednisone $5 \mathrm{mg}$ in the morning and $2.5 \mathrm{mg}$ in the evening and fludrocortisone $0.1 \mathrm{mg}$ daily since adolescence. The patient was obese and denied a history of precocious puberty, diabetes, hypertension, or excess virilization. He is married and had never attempted to have children. He reported adherence to medications but he did not recall any history of biochemical monitoring. He had not seen an endocrinologist for many years. The patient presented to his local primary care provider with chronic back pain and new lower extremity parasthesias.

\section{Investigation}

A magnetic resonance imaging (MRI) study of the lumbar spine showed large retroperitoneal masses and prompted further imaging. An abdominal CT scan showed bilateral suprarenal masses: a left $31.1 \times 18.1 \times 16.1 \mathrm{~cm}$ mass with predominant fat attenuation and a right $13.7 \times 6.6 \times$ $10.6 \mathrm{~cm}$ mass with predominant complex fat attenuation (Fig. 1). The low-density areas of the lesion measured $-97 \mathrm{HU}$, while the highest density areas measured $-5 \mathrm{HU}$. Adrenal glands were not identified. A positron emission tomography scan showed low metabolic activity in these lesions. No hormonal workup was performed by the surgical service evaluating the patient on initial presentation.

\section{Treatment}

The differential diagnosis of these masses included benign lesions such as lipoma, teratoma, or myelolipoma.

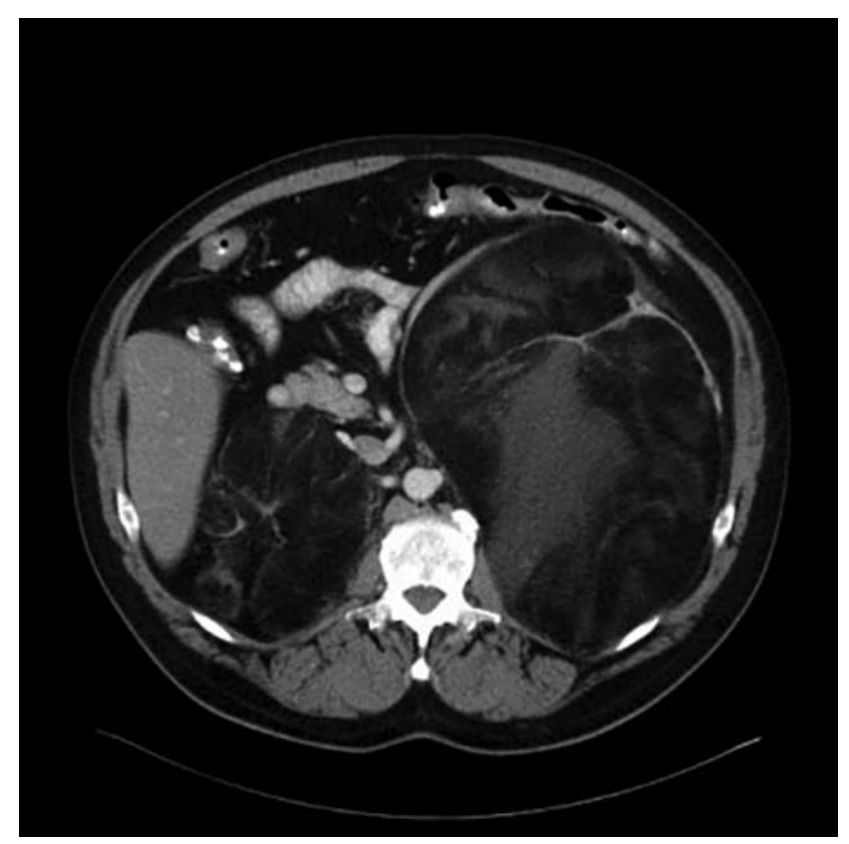

Figure 1

CT scan of abdomen showing giant bilateral myelolipomas. 


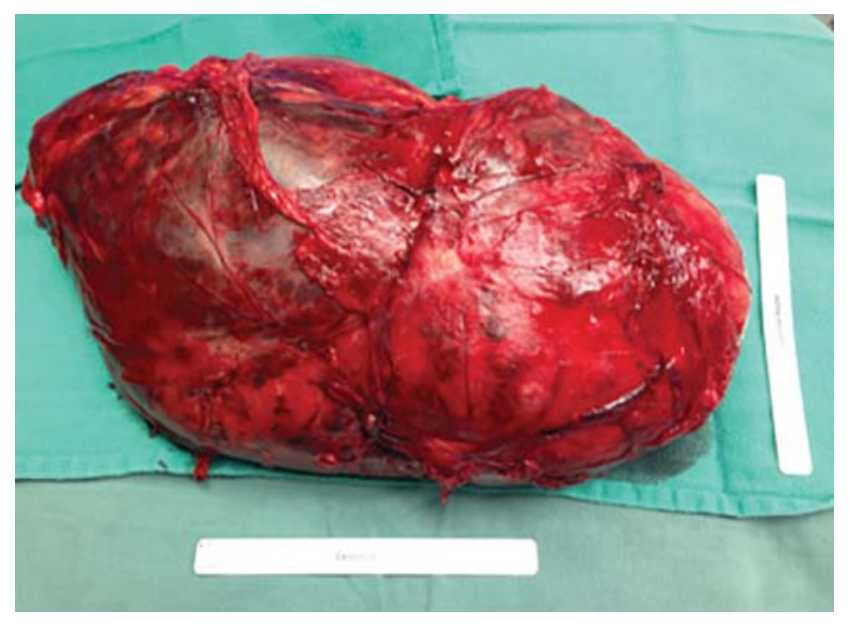

Figure 2

Gross specimen of left adrenal myelolipoma measuring $34 \times 20 \times 13 \mathrm{~cm}$.

However, due to concern for malignancy, such as liposarcoma, and symptoms from mass effect, the patient underwent bilateral resection of the retroperitoneal masses. Intraoperatively, adrenal glands were not visualized. Pathologic examination revealed fat interspersed with myeloid tissue and a diagnosis of bilateral myelolipomas was made. The left tumor was $34 \times 20 \times 13 \mathrm{~cm}$ and weighed $4.7 \mathrm{~kg}$ (Fig. 2). The right tumor measured $20 \mathrm{~cm}$ in maximal dimension and was removed in three separate fragments. Adrenal tissue was present in the both tumors (Fig. 3).

Endocrinology was consulted postoperatively. The patient had been treated with stress dose hydrocortisone perioperatively. Fludrocortisone was resumed on postoperative day 1 . His hospital course was complicated with tachycardia and hypotension, which was successfully treated with aggressive fluid resuscitation and ileus treated with bowel rest. His hydrocortisone was tapered during the stay based on his clinical status. He was discharged home on hydrocortisone $15 \mathrm{mg}$ in the morning and $10 \mathrm{mg}$ in the evening along with $0.1 \mathrm{mg}$ of fludrocortisone daily.

\section{Outcome and follow-up}

The patient had been on modestly supra-physiologic steroid replacement doses for most of his life for the management of classic salt-wasting CAH. After surgery, it was presumed that both adrenal glands were removed and suppressive doses were no longer indicated. He is currently doing well on physiologic replacement doses of hydrocortisone $10 \mathrm{mg}$ in the morning and $5 \mathrm{mg}$ in the evening and remains on the same fludrocortisone dose. His back pain has significantly improved. A repeat CT scan of the abdomen will be performed 1 year after surgery for monitoring.

\section{Discussion}

The association of myelolipomas with $\mathrm{CAH}$, Addison's disease, Nelson's syndrome, and ACTH-dependent Cushing's syndrome supports the hypothesis that excess ACTH plays a role in tumor growth. Patients with chronic inflammatory diseases, which may be associated with hypothalamic-pituitary-adrenal axis activation, had increased frequency of myelolipomas on autopsy, further supporting this theory (5). Melanocortin 2 receptor (MC2R) is a G-protein coupled receptor that is selectively activated by ACTH. Expression of the MC2R gene has been examined in myelolipomas found in $\mathrm{CAH}$ patients and in sporadic myelolipomas with variable results (7) (8). It is possible that the receptor is only expressed in the adrenocortical components within the tumor but not in the fat or myeloid tissue (8). Despite the clinical associations suggesting ACTH-mediated tumor growth, there are no consistent data to support the expression of ACTH receptor in these tumors. It is notable that in our patient, tumors developed despite compliance with modestly supraphysiologic glucocorticoid replacement. Unfortunately, we have no historical record of this patient's ACTH levels on this treatment.

Our case describes the largest reported bilateral adrenal myelolipomas in the setting of CAH. The largest

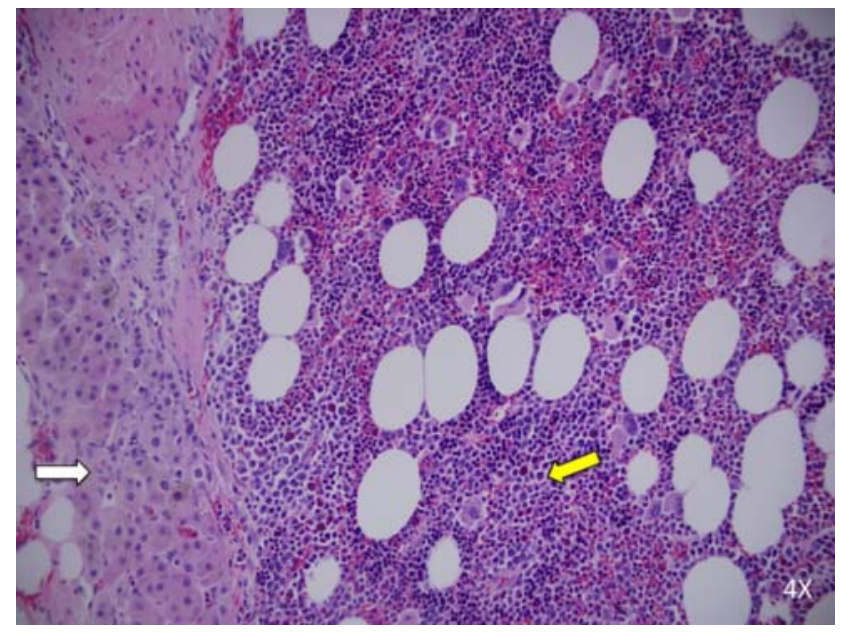

Figure 3

Histopathology (H\&E stain) of left adrenal myelolipoma showing a strip of adrenal tissue (white arrow) and myeloid issue interspersed with adipocytes (yellow arrow). 
reported unilateral myelolipoma measured was $31 \times$ $24.5 \times 11.5 \mathrm{~cm}$ and weighed $6 \mathrm{~kg}(9)$. There is varied opinion regarding size criteria for large myelolipomas as an indication for surgical resection. Many surgeons agree that tumors $>7 \mathrm{~cm}$ should be resected to avoid complications of torsion or hemorrhage and to exclude malignancy. However, if large bilateral myelolipomas are to be resected, the high likelihood of concurrent bilateral adrenalectomy should be considered and discussed with the patient and care team.

Published studies on bilateral adrenalectomy as a treatment for $\mathrm{CAH}$ to avoid iatrogenic glucocorticoid excess are limited. However, a series of 18 patients with $\mathrm{CAH}$ who underwent prophylactic bilateral adrenalectomy showed improved quality of life, lower glucocorticoid replacement doses, and decreased incidence of obesity (10). Our patient has had resolution of his mass symptoms and has also been able to decrease his maintenance glucocorticoid dose by approximately half. This case highlights the importance of considering the potential risks and benefits of planned bilateral adrenalectomy in CAH patients.

\section{Declaration of interest}

The authors declare that there is no conflict of interest that could be perceived as prejudicing the impartiality of the research reported.

\section{Funding}

This research did not receive any specific grant from any funding agency in the public, commercial or not-for-profit sector.

\section{Patient consent}

Written and informed consent has been obtained from the patient for publication of the submitted article and accompanying images.

\section{Author contribution statement}

G Kale and E M Pelley were directly involved in the care of the patient, G Kale and D B Davis conducted literature review, G Kale wrote the case report, and D B Davis and E M Pelley revised the manuscript.

\section{References}

1 Doddi S, Singhal T, Leake T \& Sinha P 2009 Management of an incidentally found large adrenal myelolipoma: a case report. Cases Journal 2 8414. (doi:10.4076/1757-1626-2-8414)

2 Daneshmand S \& Quek ML 2006 Adrenal myelolipoma: diagnosis and management. Urology Journal 3 71-74.

3 Vick CW, Zeman RK, Mannes E, Cronan JJ \& Walsh JW 1984 Adrenal myelolipoma: CT and ultrasound findings. Urologic Radiology 6 7-13. (doi:10.1007/BF02923691)

4 Nermoen I, Rorvik J, Holmedal SH, Hykkerud DL, Fougner KJ, Svartberg J, Husebye ES \& Lovas K 2011 High frequency of adrenal myelolipomas and testicular adrenal rest tumours in adult Norwegian patients with classical congenital adrenal hyperplasia because of 21-hydroxylase deficiency. Clinical Endocrinology 75 753-759. (doi:10.1111/j.1365-2265.2011.04151.x)

5 Al-Bahri S, Tariq A, Lowentritt B \& Nasrallah DV 2014 Giant bilateral adrenal myelolipoma with congenital adrenal hyperplasia. Case Reports in Surgery 2014 728198. (doi:10.1155/2014/728198)

6 German-Mena E, Zibari GB \& Levine SN 2011 Adrenal myelolipomas in patients with congenital adrenal hyperplasia: review of the literature and a case report. Endocrine Practice 17 441-447. (doi:10.4158/ EP10340.RA)

7 Almeida MQ, Kaupert LC, Brito LP, Lerario AM, Mariani BM, Ribeiro M, Monte O, Denes FT, Mendonca BB \& Bachega TA 2014 Increased expression of ACTH (MC2R) and androgen (AR) receptors in giant bilateral myelolipomas from patients with congenital adrenal hyperplasia. BMC Endocrine Disorders 14 42. (doi:10.1186/1472-6823-14-42)

8 Hagiwara H, Usui T, Kimura T, Tagami T, Naruse M, Minamiguchi S, Kato T, Okuno H \& Shimatsu A 2008 Lack of ACTH and androgen receptor expression in a giant adrenal myelolipoma associated with 21-hydroxylase deficiency. Endocrine Pathology 19 122-127. (doi:10.1007/s12022-008-9034-2)

9 Akamatsu H, Koseki M, Nakaba H, Sunada S, Ito A, Teramoto S \& Miyata M 2004 Giant adrenal myelolipoma: report of a case. Surgery Today 34 283-285. (doi:10.1007/s00595-003-2682-4)

10 Van Wyk JJ \& Ritzen EM 2003 The role of bilateral adrenalectomy in the treatment of congenital adrenal hyperplasia. Journal of Clinical Endocrinology and Metabolism 88 2993-2998. (doi:10.1210/jc.2002022026)

Received in final form 19 August 2015 Accepted 28 August 2015 